\title{
A novel function of CREG in metabolic disorders
}

https://doi.org/10.1515/mr-2021-0031

Received November 10, 2021; accepted December 13, 2021;

published online January 11, 2022

\begin{abstract}
Metabolic disorders are public health problems that require prevention and new efficient drugs for treatment. Cellular repressor of E1A-stimulated genes (CREG) is ubiquitously expressed in mature tissues and cells in mammals and plays a critical role in keeping cells or tissues in a mature, homeostatic state. Recently, CREG turns to be an important mediator in the development of metabolic disorders. Here in this review, we briefly discuss the structure and molecular regulation of CREG along with the therapeutic strategy to combat the metabolic disorders.
\end{abstract}

Keywords: CREG; insulin resistance; metabolic disorders; NAFLD; obesity; skeletal muscle energy metabolism.

\section{Introduction}

Metabolic homeostasis is important for maintaining a healthy lifespan. Due to unhealthy lifestyle behaviors such as high-calorie diet, sedentary and physical inactivity, the prevalence of metabolic disorders, including metabolic syndrome, obesity, type 2 diabetes mellitus (T2DM), nonalcoholic fatty liver disease (NAFLD) [1], poses a huge challenge to human health, which is the leading cause of global human death. There are a number of cells and molecules that contribute to altered metabolic homeostasis [2]. Out of which cellular repressor of E1A-stimulated genes (CREG) turns to be an important mediator in the development of metabolic disorders. Here in this review, we briefly discuss the structure and molecular regulation of

*Corresponding author: Yaling Han, Cardiovascular Research Institute and Department of Cardiology, General Hospital of Northern Theater Command, Shenyang 110016, Liaoning, China, E-mail: hanyaling@263.net. https://orcid.org/0000-0001-63906991

Yang Li, Xiaoxiang Tian, Quanyu Zhang and Chenghui Yan, Cardiovascular Research Institute and Department of Cardiology, General Hospital of Northern Theater Command, Shenyang 110016, Liaoning, China
CREG along with the therapeutic strategy to combat the metabolic disorders.

\section{Structure of CREG}

CREG was cloned in yeast two-hybrid screening of a Drosophila cDNA library in 1998 [3]. CREG is initially postulated as a transcription repressor for the oncoprotein E1A-activated genes, antagonizes 12SE1A-mediated transcriptional activation of both adenovirus E2 and cellular heat shock protein 70 promoters. It inhibits E1A-mediated transformation of primary cultured rat kidney cells and promotes human embryonic carcinoma cell differentiation even in the absence of an inducer such as retinoic acid $[4,5]$. Later it has been proposed that CREG acts as an extracellular ligand binding to a cell surface receptor. A functional signal sequence at its amino terminus targets the CREG protein into the endoplasmic reticulum and shows its location in the perinuclear region, a typical site for secreted proteins [4, 6]. Of the several proteins that had been reported to interact with CREG, the cationindependent mannose-6-phosphate (M6P)/insulin-like growth factor II receptor (IGF2R) has been shown to be required for its growth-suppressive activity [5]. Since this interaction was shown to depend on $\mathrm{N}$-glycosylation of CREG, it was inferred that CREG binds to M6P/IGF2R via $\mathrm{M} 6 \mathrm{P}$ residues located in its $\mathrm{N}$-glycans. Other studies have shown that CREG interacts with M6P/IGF2R in a M6Pdependent manner [7-9]. In vitro binding assay revealed that CREG bound to M6P/IGF2R extracellular domains 7-10 and 11-13 in a glycosylation-dependent and -independent manner, respectively [10]. Recently, Schahs et al. [11] revealed that CREG is a lysosomal protein that underwent proteolytic maturation in the course of its biosynthesis, carried the M6P recognition marker and depended on the interaction with M6P receptors for efficient delivery to lysosomes.

Previous studies suggest that CREG is ubiquitously expressed in mature tissues and cells in mammals and is expressed at very low levels in immature cells such as pluripotent mouse embryonic stem cells and human embryonic carcinoma cells. Induction of CREG protein and mRNA during the differentiation of these cell types have 
been investigated, with studies suggesting that CREG plays a critical role in keeping cells or tissues in a mature, homeostatic state.

\section{The role of CREG in obesity}

Obesity is one of the most common preventable diseases, which is now recognized as a major independent risk factor for metabolic diseases including insulin resistance, T2DM, coronary artery disease, stroke, NAFLD and certain cancers [12-14], as well as a disease associated with serious morbidity and increased mortality [15]. $\mathrm{CREG}^{+/-}$ mice were used to explore the role of CREG in obesity because global knockout of CREG leads to embryonic death around E7.5 (unpublished data). $\mathrm{CREG}^{+/-}$mice were viable and exhibited no apparently abnormal phenotype under normal feeding conditions, although CREG expression was reduced by $-50 \%$ in most tissues. However, when fed with high fat diet (HFD), $\mathrm{CREG}^{+-}$mice displayed significant body weight gain and visceral/subcutaneous fat adiposity compared with their wild type (WT) littermates [16]. Of note, the overt obese phenotype was not resulting from increased food intake. Additionally, the role of CREG in obesity was further investigated by creating adipocyte P2-CREG-transgenic (Tg) mice [17]. There were no phenotypic differences in body weight, food intake, and tissue weight between the WT and Tg mice under a normal feeding conditions, in which CREG mRNA level was 20-35 fold higher in each adipose tissue in the Tg mice than in the WT mice. Under the HFD condition, the Tg mice were found to be more resistant to diet-induced obesity than WT mice in the absence of a difference in food intake.

The increased adipose tissue is one of the key characteristics of obesity. Mammals have two types of adipose tissue, white adipose tissue (WAT) and brown adipose tissue (BAT), which can be distinguished by their morphology and function [18]. WAT stores excess energy as triglycerides and BAT is specialized in the dissipation of energy through the production of heat [19]. The $\mathrm{CREG}^{+/-}$ mice displayed more deposited fat in inguinal, epididymal and peri-renal WAT when fed with HFD compared to WT controls [16]. Additionally, histology of epididymal WAT showed that the size of adipocyte was larger in $\mathrm{CREG}^{+/-}$ mice than that of WT controls on HFD ( -2 folds) [17]. Nutrient overload-induced obesity is associated with a state of low grade chronic systemic inflammation predominantly resulting from adipose tissue [20]. Interestingly, CREG haploinsufficiency in the heterozygous mice amplified HFD-induced systemic as well as WAT inflammation as indicated by increased expression of cytokines and chemokines through the activation of the NF- $\mathrm{kB}$ signaling pathway, including TNF- $\alpha$, IL- 6 , and MCP-1 [16].

In contrast to WAT, BAT has entirely different functions on energy metabolism [21, 22]. Researchers have tried to transform WAT into brown to explore a new way for obesity treatment [23]. CREG exhibited the ability to stimulate brown adipogenesis, including the induction of uncoupling protein 1 (UCP1) in murine mesenchymal stem cell line C3H10T1/2 [24]. Moreover, the expression of UCP1 and fibroblast growth factor-21 and browning were both significantly higher in Tg mice than in WT littermates. It was also found that CREG binds to retinoid X receptor $\alpha$, which interacts with thyroid hormone receptor for brown adipogenesis, indicating CREG stimulates brown adipocyte formation and browning, ameliorating obesity and its related pathology in vivo [17].

In addition, age-related body weight gain was also significantly suppressed in Tg mice compared to that in WT mice. As expected, CREG levels were higher in each adipose tissue in young $\mathrm{Tg}$ mice, and this augmented expression was maintained in aged $\mathrm{Tg}$ mice compared to that in their WT littermates. Consequently, increased brown fat formation was detected in aged Tg mice, in which ageassociated metabolic phenotypes such as body weight gain were improved compared with those in WT mice under normal feeding conditions [25]. These studies suggest that CREG plays an important role in energy metabolism through the regulation of adipocyte function and contributes to the improvement of obesity and its related pathology.

\section{The role of CREG in insulin resistance}

High fat induced obesity is usually accompanied by insulin resistance and dyslipidemia [26, 27]. Obesity-induced insulin resistance is one of the largest noncommunicable disease epidemics that we are facing at the moment [28]. Insulin resistance is the condition where the body does not respond appropriately to circulating insulin [29, 30]. Insulin resistance occurs in several tissues, including the liver, muscle and adipose tissue. The liver helps to maintain fasting glucose levels through gluconeogenesis and glycogenolysis. However, when the liver is insulinresistant, the suppression of hepatic glucose production is impaired and thus gluconeogenesis and glycogenolysis continue at inappropriately high levels despite normal or high circulating glucose levels. Adipose tissue and muscle are similarly affected by insulin resistance, although the problem here relates more to the impaired ability of insulin 
to promote glucose disposal [29]. To compensate for the insulin resistance in these tissues, pancreatic $\beta$-cells produce more insulin. However, there is a limit to how much can be produced, and when this has been reached, the $\beta$-cells fail. T2DM occurs when an inappropriately low level of insulin is produced in response to a given concentration of glucose.

Blood glucose and insulin levels of $\mathrm{CREG}^{+/-}$mice were similar to those of WT controls under normal diet. It's not surprisingly to see that the glucose level in $\mathrm{CREG}^{+/-}$mice was significantly higher when fed with HFD, beginning as early as 4 weeks of HFD feeding and continuing to the end of the experiment, and so was the plasma insulin level as compared with WT controls. Integrated plasma glucose concentration, as calculated by AUC, was more profoundly increased in $\mathrm{CREG}^{+/-}$mice as compared with WT controls on HFD but not normal diet [16]. However, the adipocyte and hepatocyte specific CREG Tg mice reversed the high blood glucose and high serum insulin levels induced by HFD feeding, as well as exhibited an improvement of insulin tolerance compared with WT mice as assessed by glucose tolerance tests, insulin tolerance tests, and insulin signaling analysis [17, 31].

There is increasing evidence showing that inflammation is an important pathogenic mediator of the development of obesity-induced insulin resistance [32]. The plasma level of the pro-inflammatory cytokines (TNF- $\alpha$ and IL-6), the pro-inflammatory adipokine leptin and the antiinflammatory adipokine adiponectin in $\mathrm{CREG}^{+/-}$mice was similar to that in the WT control on ND. The pro-inflammatory chemokine MCP-1 showed a tendency of increase in $\mathrm{CREG}^{+/-}$ mice but failed to reach significance, suggesting that reduction of CREG may affect some cytokine expression even on a normal diet. By contrast, on HFD, $\mathrm{CREG}^{+/-}$mice had significantly higher levels of tumor necrosis factor $-\alpha$, interleukin- 6 , monocyte chemoattractant protein-1, leptin and a lower level of adiponectin in plasma [16]. Furthermore, CREG haploinsufficiency caused a more robust, 2-fold increase of p-NF-kB (p65) as compared with the WT control on HFD [16], indicating that CREG haploinsufficiency increases HFDinduced systemic inflammation, which may be closely related to insulin resistance. In addition, the benefits provided by CREG-induced expression of fibroblast growth factor 21 are showing promise in pharmacological application for improving insulin resistance $[33,34]$.

\section{The role of CREG in NAFLD}

NAFLD represents a spectrum of conditions ranging from increased intrahepatic accumulation of triglyceride, that is fatty liver aka hepatic steatosis, to non-alcoholic steatohepatitis, a state of hepatocellular inflammation and ballooning with possible collagen deposition, which can progress further to fibrosis, cirrhosis and hepatocellular carcinoma [35]. NAFLD affects $25 \%$ of the world's population [36] and increases in line with obesity and T2DM, and there is no approved drug therapy [37].

The elevation of plasma total cholesterol, triglyceride, low density lipoprotein-cholesterol and free fatty acid in $\mathrm{CREG}^{+/-}$mice suggested dysfunction in lipid metabolism, possibly resulting from abnormalities of WAT and/or the liver [16]. In a mouse NAFLD model, the mRNA and protein expression levels of CREG were clearly decreased after being fed an HFD for 12 weeks compared with those of the normal diet-treated control mice. Hepatocyte-specific CREG deletion dramatically exacerbates HFD and leptin deficiency-induced hepatic steatosis, whereas a beneficial effect is conferred by CREG overexpression [31]. Mechanistically, CREG interacts directly with apoptosis signalregulating kinase 1 and inhibits its phosphorylation, thereby blocking the downstream MAPK kinase 4/7-Jun $\mathrm{N}$-terminal kinase 1 signaling pathway and leading to significantly alleviated hepatic steatosis [31], suggesting that CREG might be a promising therapeutic target for NAFLD and related metabolic diseases.

\section{The role of CREG in skeletal muscle energy metabolism}

Skeletal muscle is the largest metabolic organ in the human body, consuming about $18 \%$ of the entire body daily expenditure of energy [38]. It produces various secrete factors and participates in the interplay among multiple tissues and organs [39, 40]. Loss of muscle mass or strength due to aging or diseases may result in decreased physical activity and elevated risks for cardiovascular diseases, T2DM, and cancer [41]. Conversely, maintaining skeletal muscle mass might be effective in the prevention and treatment of T2DM, cardiovascular diseases and cancer/ cachexia [42]. Skeletal muscle oxidative metabolism in vivo may be lowered by hypoxia because of reduced perfusion in obesity, which may precede loss in muscle mitochondrial density and promote glycolytic energy utilization [43]. Therefore, changes in control of skeletal muscle circulation and vascular dysfunction that occur with obesity may be implicated in impairment of skeletal muscle energy metabolism [44].

The recent research showed CREG was localized to the mitochondria both in vivo and in vitro and played an 
important role in mitophagy. In skeletal muscle-specific CREG knockout mouse model, the exercise time to exhaustion and running distance were significantly reduced [45], a phenotype explained by mitophagy impairment. In addition, the administration of recombinant CREG protein improved the motor function. Mechanistically, CREG mediated the effect of HSPD1, a mitochondrial chaperon protein, the instability of which resulted in increased mitophagy and muscle dysfunction. CREG deficiency accelerated the induction of mitophagy in the skeletal muscle. Furthermore, HSPD1/HSP60 (heat shock protein 1) (401-573 aa) interacted with CREG (130-220 aa) to antagonize the degradation of CREG and was involved in the regulation of mitophagy [45].

\section{Summary}

This review highlights the diverse biological actions of CREG, as well as the therapeutic potential for metabolic disorders. Future studies are required to fully understand the signaling pathways and mechanisms involved in the functions of CREG in obesity, insulin resistance, NAFLD, as well as skeletal muscle energy metabolism. Nevertheless, CREG possesses a promising therapeutic repertoire that may result in the development of a safe, effective, longacting and cost-effective therapy for metabolic disorders.

Research funding: The study was supported by Key R\&D Program of Liaoning Province of China (2020JH 2/10300167) and Cardiacare Sponsored Optimizing Antithrombotic Research Fund of China (BJUHFCSOARF201901-06).

Author contributions: All authors have accepted responsibility for the entire content of this manuscript and approved its submission.

Competing interests: Authors state no conflict of interest. Informed consent: Informed consent was obtained from all individuals included in this study.

Ethical approval: The local Institutional Review Board deemed the study exempt from review.

\section{References}

1. Su ZQ, Guo YR, Huang XF, Feng B, Tang LP, Zheng GJ, et al. Phytochemicals: targeting mitophagy to treat metabolic disorders. Front Cell Dev Biol 2021;9:686820.

2. Garg R, Kumariya S, Katekar R, Verma S, Goand UK, Gayen JR. JNK signaling pathway in metabolic disorders: an emerging therapeutic target. Eur J Pharmacol 2021;901:174079.

3. Veal E, Eisenstein M, Tseng ZH, Gill G. A cellular repressor of E1A-stimulated genes that inhibits activation by E2F. Mol Cell Biol. 1998;18:5032-41.
4. Veal E, Groisman R, Eisenstein M, Gill G. The secreted glycoprotein CREG enhances differentiation of NTERA-2 human embryonal carcinoma cells. Oncogene 2000;19:2120-8.

5. Di Bacco A, Gill G. The secreted glycoprotein CREG inhibits cell growth dependent on the mannose-6-phosphate/ insulin-like growth factor II receptor. Oncogene 2003;22: 5436-45.

6. Li J, Han YL, Yan CH, Kang J, Peng CF, Zhang N, et al. A novel method to inhibit apoptosis and promote differentiation of induced pluripotent stem cells in transplantation therapy for myocardial infarction. Med Hypotheses 2011;76:264-5.

7. Journet A, Chapel A, Kieffer S, Louwagie M, Luche S, Garin J. Towards a human repertoire of monocytic lysosomal proteins. Electrophoresis 2000;21:3411-9.

8. Journet A, Chapel A, Kieffer S, Roux F, Garin J. Proteomic analysis of human lysosomes: application to monocytic and breast cancer cells. Proteomics 2002;2:1026-40.

9. Sleat DE, Lackland H, Wang Y, Sohar I, Xiao G, Li H, et al. The human brain mannose 6-phosphate glycoproteome: a complex mixture composed of multiple isoforms of many soluble lysosomal proteins. Proteomics 2005;5:1520-32.

10. Han YL, Luan B, Sun MY, Guo L, Guo P, Tao J, et al. Glycosylationindependent binding to extracellular domains 11-13 of mannose-6-phosphate/insulin-like growth factor-2 receptor mediates the effects of soluble CREG on the phenotypic modulation of vascular smooth muscle cells. J Mol Cell Cardiol 2011;50:723-30.

11. Schähs P, Weidinger P, Probst OC, Svoboda B, Stadlmann J, Beug $\mathrm{H}$, et al. Cellular repressor of E1A-stimulated genes is a bona fide lysosomal protein which undergoes proteolytic maturation during its biosynthesis. Exp Cell Res 2008;314: 3036-47.

12. Klil-Drori AJ, Azoulay L, Pollak MN. Cancer, obesity, diabetes, and antidiabetic drugs: is the fog clearing? Nat Rev Clin Oncol. 2017; 14:85-99.

13. Mitchell AB, Cole JW, McArdle PF, Cheng YC, Ryan KA, Sparks MJ, et al. Obesity increases risk of ischemic stroke in young adults. Stroke 2015;46:1690-2.

14. Csige I, Ujvárosy D, Szabó Z, Lőrincz I, Paragh G, Harangi M, et al. The impact of obesity on the cardiovascular system. J Diabetes Res 2018;2018:3407306.

15. Lingvay I, Sumithran P, Cohen RV, le Roux CW. Obesity management as a primary treatment goal for type 2 diabetes: time to reframe the conversation. Lancet 2021;30:S01406736(21)01919-X.

16. Tian X, Yan C, Liu M, Zhang Q, Liu D, Liu Y, et al. CREG1 heterozygous mice are susceptible to high fat diet-induced obesity and insulin resistance. PLoS One 2017;12:e0176873.

17. Hashimoto M, Kusudo T, Takeuchi T, Kataoka N, Mukai T, Yamashita H. CREG1 stimulates brown adipocyte formation and ameliorates diet-induced obesity in mice. FASEB J 2019;33: 8069-82.

18. Okita N, Hayashida Y, Kojima Y, Fukushima M, Yuguchi K, Mikami K, et al. Differential responses of white adipose tissue and brown adipose tissue to caloric restriction in rats. Mech Ageing Dev 2012;133:255-66.

19. Saely $\mathrm{CH}$, Geiger K, Drexel H. Brown versus white adipose tissue: a mini-review. Gerontology 2012;58:15-23.

20. van der Heijden RA, Sheedfar F, Morrison MC, Hommelberg PP, Kor D, Kloosterhuis NJ, et al. High-fat diet induced obesity primes 
inflammation in adipose tissue prior to liver in C57BL/6j mice. Aging (Albany NY) 2015;7:256-68.

21. Shinde $A B$, Song $A$, Wang $Q A$. Brown adipose tissue heterogeneity, energy metabolism, and beyond. Front Endocrinol (Lausanne) 2021;12:651763.

22. Gesta S, Tseng YH, Kahn CR. Developmental origin of fat: tracking obesity to its source. Cell 2007;131:242-56.

23. Qian S, Tang Y, Tang QQ. Adipose tissue plasticity and the pleiotropic roles of BMP signaling. J Biol Chem 2021;296:100678.

24. Kusudo T, Hashimoto M, Kataoka N, Li Y, Nozaki A, Yamashita H. CREG1 promotes uncoupling protein 1 expression and brown adipogenesis in vitro. J Biochem 2019;165:47-55.

25. Hashimoto M, Goto A, Endo Y, Sugimoto M, Ueda J, Yamashita H. Effects of CREG1 on age-associated metabolic phenotypes and renal senescence in mice. Int J Mol Sci 2021;22:1275.

26. Guo R, Zhang Y, Turdi S, Ren J. Adiponectin knockout accentuates high fat diet-induced obesity and cardiac dysfunction: role of autophagy. Biochim Biophys Acta 2013; 1832:1136-48.

27. Zhang Y, Yuan M, Bradley KM, Dong F, Anversa P, Ren J. Insulinlike growth factor 1 alleviates high-fat diet-induced myocardial contractile dysfunction: role of insulin signaling and mitochondrial function. Hypertension 2012;59:680-93.

28. Prasad M, Chen EW, Toh SA, Gascoigne NRJ. Autoimmune responses and inflammation in type 2 diabetes. J Leukoc Biol 2020;107:739-48.

29. Lee BC, Lee J. Cellular and molecular players in adipose tissue inflammation in the development of obesity-induced insulin resistance. Biochim Biophys Acta 2014;1842:446-62.

30. Kahn SE, Hull RL, Utzschneider KM. Mechanisms linking obesity to insulin resistance and type 2 diabetes. Nature 2006;444: 840-6.

31. Zhang QY, Zhao LP, Tian XX, Yan CH, Li Y, Liu YX, et al. The novel intracellular protein CREG inhibits hepatic steatosis, obesity, and insulin resistance. Hepatology 2017;66:834-54.

32. SantaCruz-Calvo S, Bharath L, Pugh G, SantaCruz-Calvo L, Lenin R, Lutshumba J, et al. Adaptive immune cells shape obesityassociated type 2 diabetes mellitus and less prominent comorbidities. Nat Rev Endocrinol 2022;18:23-42.
33. Ni B, Farrar JS, Vaitkus JA, Celi FS. Metabolic effects of FGF-21: thermoregulation and beyond. Front Endocrinol (Lausanne) 2015; 6:148.

34. Fisher FM, Maratos-Flier E. Understanding the physiology of FGF21. Annu Rev Physiol 2016;78:223-41.

35. Tiniakos DG, Vos MB, Brunt EM. Nonalcoholic fatty liver disease: pathology and pathogenesis. Annu Rev Pathol 2010;5:145-71.

36. Younossi Z, Tacke F, Arrese M, Chander Sharma B, Mostafa I, Bugianesi $\mathrm{E}$, et al. Global perspectives on nonalcoholic fatty liver disease and nonalcoholic steatohepatitis. Hepatology 2019;69: 2672-82.

37. Mitrovic M, Sistilli G, Horakova O, Rossmeisl M. Omega-3 phospholipids and obesity-associated NAFLD: potential mechanisms and therapeutic perspectives. Eur J Clin Invest 2021: e13650.

38. Tian H, Liu S, Ren J, Lee JKW, Wang R, Chen P. Role of histone deacetylases in skeletal muscle physiology and systemic energy homeostasis: implications for metabolic diseases and therapy. Front Physiol 2020;11:949.

39. Mizgier ML, Fernández-Verdejo R, Cherfan J, Pinget M, Bouzakri K, Galgani JE. Insights on the role of putative muscle-derived factors on pancreatic beta cell function. Front Physiol 2019;10:1024.

40. Pedersen BK, Febbraio MA. Muscles, exercise and obesity: skeletal muscle as a secretory organ. Nat Rev Endocrinol 2012;8:457-65.

41. Balan E, Schwalm C, Naslain D, Nielens H, Francaux M, Deldicque L. Regular endurance exercise promotes fission, mitophagy, and oxidative phosphorylation in human skeletal muscle independently of age. Front Physiol 2019;10:1088.

42. Zieff GH, Wagoner CW, Paterson C, Lassalle PP, Lee JT. Cardiovascular consequences of skeletal muscle impairments in breast cancer. Sports (Basel) 2020;8:80.

43. Stapleton PA, James ME, Goodwill AG, Frisbee JC. Obesity and vascular dysfunction. Pathophysiology 2008;15:79-89.

44. Limberg JK, Morgan BJ, Schrage WG. Peripheral blood flow regulation in human obesity and metabolic syndrome. Exerc Sport Sci Rev 2016;44:116-22.

45. Song H, Tian X, Liu D, Liu M, Liu Y, Liu J, et al. CREG1 improves the capacity of the skeletal muscle response to exercise endurance via modulation of mitophagy. Autophagy 2021;17:4102-18. 